for $i, j, k=1,2, \cdots, r$, are the structural constants of $G$, we have $c_{i j k}+c_{i k j}=0$.

F. N. Cole,

Secretary.

\title{
COMPLETE EXISTENTIAL THEORY OF THE POSTULATES FOR SERIAL ORDER.
}

\author{
BY PROFESSOR EDWARD V. HUNTINGTON.
}

(Read before the American Mathematical Society, December 27, 1916.)

The purpose of this note is to establish the " complete independence"-in the sense defined by E. H. Moore*-of each of three different sets of postulates for serial order.

The first set of postulates ( more convenient for many purposes than either of the other sets. Set $B$ dates back to Vailati, 1892. $\dagger$ Set $C$ (a modification of set $B$ and now widely used) was introduced by the present writer in $1905 . \$$

The universe of discourse considered in each of these sets is the universe of all systems $(K, R)$, in which $K$ is a class of elements, $A, B, C, \cdots$, and $R$ is a dyadic relation; the notation $R(A B)$, or briefly $A B$, meaning that the relation $R$ holds

* E. H. Moore, "Introduction to a form of general analysis," Yale University Press (1910), p. 82. An interesting example of a proof of complete independence is given by R. D. Beetle, "On the complete independence of Schimmack's postulates for the arithmetic mean," Math. Annalen, vol. 76 (1915), pp. 444-446. [Compare R. Schimmack, "Der Satz vom arithmetischen Mittel in axiomatischer Begründung," Math. Annalen, vol. 68 (1909), pp. 125-132.] For a similar discussion of an almost completely independent set of postulates, see L. I. Dines, "Complete existential theory of Sheffer's pcstulates for Boolean algebras," this Bulletin, vol. 21 (1915), pp. 183-188. [Compare H. M. Sheffer, "A set of five independent postulates for Boolean algebras, with application to logical constants," Transactions Amer. Math. Society, vol. 14 (1913), pp. 481-488.]

† G. Vailati, "Sui principî fondamentali della geometria della retta," Rivista di Matematica, vol. 2 (1892), pp. 71-75; B. Russell, Principles of Mathematiss, vol. 1 (1903), pp. 203, 218-219.

$\ddagger$ E. V. Huntington, "The continuum as a type of order," reprinted from the Annals of Mathematics, vols. 6 and 7 (1905), especially vol. 6, pp. 157-158; second edition, Harvard University Press, 1917, pp. 10-11, J. W. Young, Fundamental Concepts of Algebra and Geometry (1911), p. 68; A. N. Whitehead and B. Russell, Principia Mathematica, vol. 2 (1912), p. 513. (In the present terminology of Whitehead and Russell, a relation which satisfies postulate 1 is said to be "contained in diversity."). 
for the elements $A$ and $B$, in the order stated. The "postulates for serial order" are the conditions which such a system $(K, R)$ must satisfy in order to be called a " serial system." If these conditions are satisfied, the relation $R$ is called a serial relation with respect to the class $K$, and the notation $R(A B)$, or $A B$, may then be replaced by the more familiar notation $A<B$ (" $A$ precedes $B$ ").

\section{Set A. (Postulates 1, 2, 3, 4.)}

The first postulate in this set concerns only a single element; the next two concern two distinct elements; and the fourth concerns three distinct elements.

Postulate 1. $A A .=.0$.

(Irreflexiveness.)

That is, if $A$ is an element of the class $K$, then the statement $A A$ is always false.

Postulate 2. $A \neq B: \supset: A B \cup B A$. (Connexity.)

That is, if $A$ and $B$ are distinct elements of $K$, then at least one of the statements $A B$ and $B A$ will be true.

Postulate 3. $A \neq B . A B . B A .=.0$.

(Asymmetry for distinct elements.)

That is, if $A$ and $B$ are distinct elements of $K$, then not both the statements $A B$ and $B A$ can be true.

Postulate 4. $A \neq B . A \neq C . B \neq C . A B . B C: \supset: A C$. (Transitivity for distinct elements.)

That is, if $A, B$, and $C$ are distinct elements of $K$, then $A B$ and $B C$ together imply $A C$.

\section{Set B. (Postulates 2, 3a, 4a.)}

This set contains only three postulates, $3 a$ and $4 a$ being more inclusive forms of 3 and 4 .

Postulate 2. $A \neq B: \supset: A B \cup B A$.

Postulate $3 a$. AB.BA:=: 0 .

(Connexity.)

(Asymmetry for all elements.)

That is, if $A$ and $B$ are any elements of $K$ (whether distinct or not), then not both the statements $A B$ and $B A$ can be true.

Postulate 4a. $A B . B C: \supset: A C$.

(Transitivity for all elements.)

That is, if $A$ and $B$ are any elements of $K$ (whether distinct or not), then $A B$ and $B C$ together imply $A C$. 
Here $3 a$ implies 3 and 1 , and $4 a$ implies 4 ; also 1 and 3 imply $3 a$, and 4 and 3 imply $4 a$; so that sets $A$ and $B$ are clearly equivalent.

Note also that in set $B, 4 a$ may be replaced by 4 , giving another set, $B^{\prime}$, comprising postulates $2,3 a$, and 4 .

\section{Set C. (Postulates 1, 2, 4a.)}

This set is a little simpler than set $B$, since postulate 1 is a little simpler than postulate $3 a$; but neither set $B$ nor set $C$ is as explicit as $\operatorname{set} A$.

Postulate 1. $A A .=.0$.

(Irreflexiveness.)

Postulate 2. $A \neq B: \supset: A B \cup B A$.

(Connexity.)

Postulate 4a. $A B . B C: \supset: A C$.

(Transitivity for all elements.)

Here 1 and $4 a$ imply $3 a$; and $3 a$ implies 1 ; so that sets $B$ and $C$ are equivalent.

The question of the "complete independence" of each of these sets is in reality a question of classification. Every system $(K, R)$, in the universe considered, either satisfies or fails to satisfy each of the postulates of each set. Now set $A$ contains four postulates, and sets $B$ and $C$ each contain three. Hence set $A$ divides the universe theoretically into 16 compartments, while each of the sets $B$ and $C$ divides it into 8 compartments. The purpose of this note is to show that no one of these compartments is "empty"; that is, to show that all the types of system $(K, R)$ which can be distinguished by means of the postulates of any one of these three sets are actually represented among existing systems.

To prove this for set $A$, we exhibit 16 examples of systems $(K, R)$; the first 8 of these answer also for sets $B$ and $C$. In each of these examples, the class $K$ is supposed to consist of only three elements, marked $1,2,3$; the relation $R$ is defined in each case by tabulating all the dyadic statements that are supposed to be true in that case. The character of each example is then shown in the table below, in which a dot (.) indicates that a postulate holds, and a cross $(\mathrm{X})$ that it fails.

An examination of this table shows that examples of all the required types exist. (The entries below the double line are not necessary for the proof.)

In each of the examples shown, the class $K$ consists of only 
TABLe I.

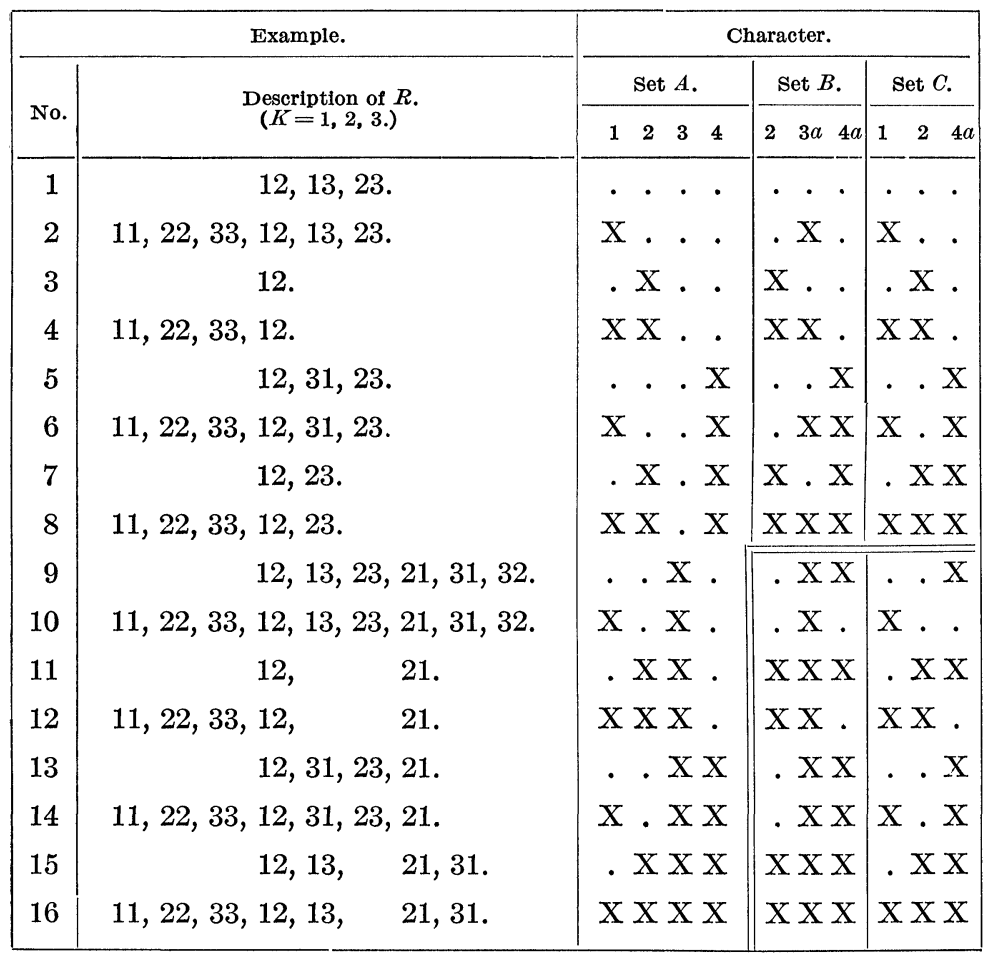

three elements. It is easy to construct similar examples, however, in which $K$ contains any number of elements, marked $1,2,3, \cdots n$, where $n$ may be either finite $(n>3)$, or denumerably infinite. This may be done by adding the following items to the " descriptions of $R$ " in the examples given above: in the examples which satisfy postulate 2 , add $x y$ whenever either $x$ or $y$ is $>3$ and $x<y$; in examples 9 and 10 add also $x y$ whenever either $x$ or $y$ is $>3$ and $x>y$; in each of the even-numbered examples, add $x x$ whenever $x>3$. The character of these modified examples remains unchanged.

If we desire a categorical set of postulates for a finite series, we have merely to add to any one of our sets of postulates a postulate demanding that the system $(K, R)$ shall contain exactly $n$ elements $(n>3)$. The modified examples just 
described show that each such categorical set of postulates will be also " completely independent."

HaRVARD UNIVERSITY.

\title{
COMPLETE EXISTENTIAL THEORY OF THE POSTU-
} LATES FOR WELL ORDERED SETS.

\author{
BY PROFESSOR EDWARD V. HUNTINGTON.
}

(Read before the American Mathematical Socjety, December 27, 1916.)

A system $(K, R)$, where $K$ is a class of elements $A, B, C, \cdots$ and $R$ is a dyadic relation, is called a well ordered system when the following conditions are satisfied:*

(a) the system $(K, R)$ is a series; and

(b) every subsystem of $(K, R)$ has a leading element. $\dagger$

Now when condition $(b)$ is added to the conditions $(a)$ which define a series, some of the conditions $(a)$ become redundant. After eliminating these redundancies, we find the following three sets of independent postulates for well ordered systems, each of these three sets being in fact " completely independent" in the sense of E. H. Moore. (The numbering of the postulates is made to conform with that in the preceding note.)

\section{Set I. (Postulates $1,3,5$. )}

Postulate 1. $A A .=.0$.

(Irreflexiveness.)

Postulate 3. $A \neq B . A B . B A:=: 0$.

(Asymmetry for distinct elements.)

Postulate 5. Every subsystem has at least one leading element.

("Leadership," or the property of being "supplied with leaders.")

* G. Cantor, Math. Annalen, vol. 49 (1897), p. 208. A. N. Whitehead and B. Russell, Principia Mathematica, vol. 3 (1913), p. 4.

$\dagger$ Here by a series we understand any system $(K, R)$ which satisfies any one of the sets of postulates mentioned in the preceding note. A subsystem of $(K, R)$ means any system $\left(K^{\prime}, R^{\prime}\right)$ such that $K^{\prime}$ is a subclass of $K$, and $R^{\prime}=R$. (Here $K^{\prime}$ is called a subclass of $K$ if every element of $K^{\prime}$ belongs to $K$; that is, a subclass is either a part or the whole.) A leading element of a system means any element $X$ having the following property: whenever $Y$ is any other element of the system, then $R(X Y)$, or simply $X Y$, will be true. (If a system contains only a single element $X$, then $X$ is a leading element of that system.) 